\title{
Multivariable Analysis of Risk Factors Affecting Dislocation After Bipolar Hemiarthroplasty in Patients with Femoral Neck Fracture
}

\author{
Yuhui Yang ${ }^{1, *}$, Guangtao Fu $\mathbb{D}^{1}{ }^{1}$, , Qingtian $\mathrm{Li}^{1}{ }^{*}$, Ruiying Zhang', Weihong Liao',2, Yuanchen Ma', \\ Qiujian Zheng ${ }^{1,2}$ \\ 'Department of Orthopedics, Guangdong Provincial People's Hospital, Guangdong Academy of Medical Sciences, Guangzhou, 5 I0080, People's \\ Republic of China; ${ }^{2}$ The Second School of Clinical Medicine, Southern Medical University, Guangzhou, 5105I5, People's Republic of China \\ *These authors contributed equally to this work \\ Correspondence: Yuanchen Ma; Qiujian Zheng, Email mayuanchen@gdph.org.cn; zhengqiujian@gdph.org.cn
}

Objective: This study aimed to investigate the risk factors associated with dislocation and dissociation following bipolar hemiarthroplasty (HA) for the treatment of patients with femoral neck fractures.

Methods: We retrospectively reviewed 462 patients (479 hips) treated with bipolar HA from January 2010 to January 2020 . All patients received posterolateral approaches and a minimum follow-up of at least 2 years regularly. A case-control study was performed to analyze the risk factors of dislocation regarding patient demographics, coexisting diseases, surgical and morphologic features. Multivariable logistic regression analysis for independent risk factors affecting dislocation and dissociation was also performed.

Results: The dislocation rate was 5.01\%, and the mean time from HA to the first incident of dislocation was 38.75 days. Patientrelated factors, including operation side, prosthesis type, and neuromuscular disease, did not differ significantly. Regarding the morphological factors, a significant difference was observed in center-edge (CE) angle, abduction angle, acetabular depth, depth/ width ratio, rotation center (RC) to greater trochanter tip (GTT) vertical distance, RC to GTT vertical distance difference, RC to GTT horizontal distance, RC to GTT horizontal distance difference, offset difference, and offset discrepancy. Further, decreased CE angle, increased abduction angle, decreased RC to GTT vertical distance, decreased offset difference and increased offset discrepancy were determined to be independent risk factors of dislocation. The proportion of patients experiencing dissociation was $1.04 \%$. All the implanted femoral heads were smaller than $43 \mathrm{~mm}$, which was determined to be the risk factor of dissociation.

Conclusion: Decreased CE angle, RC to GTT vertical distance, offset difference, and increased abduction angle, offset discrepancy were determined to be independent risk factors of HA dislocation. Once dislocation risk was detected by simulated templating, THA or changing surgical approach should be considered to avoid evitable perioperative complications.

Keywords: bipolar hemiarthroplasty, dislocation, morphological evaluation, greater trochanter tip, offset

\section{Introduction}

Globally, hip fractures are a leading cause of disability in adults. Bipolar hemiarthroplasty (HA) is considered as the standard surgical procedure for elderly patients with displaced femoral neck fracture due to its relatively short operating time and prompt recovery. ${ }^{1-3}$ The surgical technique led to improved functional outcomes and decreased mortality during the perioperative period. Compared with THA, bipolar hemiarthroplasty has the advantages of a lower economic burden and a lower risk of dislocation after surgery. ${ }^{4,5}$ Pooled estimates from randomized, controlled trials suggested a risk of dislocation after total hip arthroplasty that is more than 2.5 times as great as that associated with hemiarthroplasty for hip fracture patients. ${ }^{6}$

As a relatively rare but severe complication, dislocation still turns out to be the most common reason for the revision after $\mathrm{HA},{ }^{7}$ with rates reported varying between $0.8 \%$ and $6.1 \% .{ }^{8-10}$ Accordingly, dislocation as a reason for revision was 
more common in younger patients, ASA III/IV patients, and a posterolateral approach. ${ }^{7}$ Treating these elderly patients is challenging due to their frailty and frequent multiple comorbidities. Once dislocation occurs, the management of patients is fraught with difficulty due to frailty, comorbidity, and poor quality of bone and soft tissues. Dislocation results in higher morbidity, increased financial cost and hospital stay length. ${ }^{8,11}$

Several studies have identified risk factors for dislocation in patients after undergoing hip HA. ${ }^{12-14}$ However, conclusive evidence is rare and some studies have presented contradicting results. Generally, risk factors were classified into patient factors, surgical factors, and morphological factors. Patient-related risk factors were mainly associated with neuromuscular disorders, including Dementia, Parkinson's disease, and cerebral infarction. ${ }^{9}$ Surgery factors were associated with different surgical approaches and prosthetic types. ${ }^{15}$ Morphological factors including femoral head size, femoral offset, leg-length discrepancy, acetabular center edge (CE) angle and coverage ratio have been identified as potential factors affecting the dislocation rates. ${ }^{13-16}$ However, a systematic and comprehensive evaluation was not performed. Dissociation, as a special type of dislocation, is defined as the separation of bipolar components during dislocation. We found little information regarding this rare and severe complication except a few case reports. ${ }^{17-19}$

In this study, we aimed to investigate risk factors that may cause dislocation and dissociation following a bipolar hemiarthroplasty in patients with femoral neck fracture by evaluating the morphological features in addition to the patient characteristics. Further, the risk factors determined by multivariable analysis were also discussed according to the morphometric differences between dislocated and matched control patients.

\section{Materials and Methods Study Population}

The study was approved by the institutional review board of Guangdong Provincial People's Hospital. We retrospectively reviewed 462 patients (479 hips) treated with bipolar hemiarthroplasty for femoral neck fracture from January 2010 to January 2020. All patients received posterolateral approaches and a minimum follow-up of at least 2 years regularly. Patients treated using other approaches, with pathological fractures, trochanteric fractures, previous hip fractures, surgical history and less than 2-year follow-up were excluded. Totally, we identified 24 patients who sustained one or more dislocations. Among them, one patient with substandard radiographs and three patients were lost to follow-up. Herein, 20 patients met the inclusion criteria and were included in the dislocation group, whose characteristics and medical intervention are detailed in Table 1. A matched control group of 100 patients (ratio of 1:5) without dislocation was selected from the remaining patients based on age, sex, and time of primary arthroplasty.

\section{Medical Procedure}

All the surgical operations were performed through a posterolateral approach in the lateral decubitus position. Short external rotator tendons and posterior joint capsules were repaired in all cases. After surgery, standard hip anteroposterior and lateral radiographs were taken, and full weight-bearing exercises were allowed using crutches within 48 hours postoperatively. All patients were advised to avoid $>90^{\circ}$ of flexion, excessive adduction, and internal rotation in hip movements. Demographic data, operative records and coexisting diseases for the subjects were collected from patient medical records.

\section{Image Analysis and Measurements}

$\mathrm{X}$-ray evaluation procedure was performed by two analysts blindly, without knowing the medical records and group assignment. Morphological evaluation, including acetabular measurements, rotation center measurements and femoral measurements were performed from the hip anteroposterior radiographs after surgery. Regarding the acetabular measurements, the femoral head coverage ratio was first measured after determining the lateral edge of the bipolar femoral head and acetabulum (Figure 1A). Before measurement, radiographic magnification was adjusted using the diameter of the prosthesis. Acetabular measurements also contained center-edge (CE) angle, abduction angle, acetabular depth, acetabular width, and the $\mathrm{D} / \mathrm{W}$ ratio (Figure 1B). The interteardrop line was defined as the horizontal reference line. Based on the bilateral rotation center (RC) and greater trochanter tip (GTT), rotation center measurements included femoral head diameter difference, RC 
Table I Dislocation Patient Demographics

\begin{tabular}{|c|c|c|c|c|c|c|}
\hline $\begin{array}{l}\text { Patient } \\
\text { Number }\end{array}$ & Sex & Age (Years) & $\begin{array}{l}\text { Dislocation Time } \\
\text { from Surgery } \\
\text { (Days) }\end{array}$ & $\begin{array}{l}\text { Mechanism of } \\
\text { Dislocation }\end{array}$ & Final Treatment & Coexisting Disease \\
\hline 1 & Female & 80 & 46 & Atraumatic & Closed reduction & Parkinson and dementia \\
\hline 2 & Male & 82 & 340 & Fall & Open reduction & No \\
\hline 3 & Male & 84 & 40 & Atraumatic & Open reduction & No \\
\hline $4^{*}$ & Female & 76 & 14 & Atraumatic & Open reduction & Cerebral infarction \\
\hline 5 & Female & 91 & 53 & Atraumatic (BRI) & Closed reduction & No \\
\hline 6 & Female & 75 & 15 & Atraumatic (BRI) & Open reduction & No \\
\hline 7 & Female & 92 & 8 & Atraumatic (BRI) & THA & No \\
\hline 8 & Female & 78 & 9 & Atraumatic & Closed reduction & No \\
\hline 9 & Male & 80 & 3 & Atraumatic & Closed reduction & Parkinson \\
\hline 10 & Female & 93 & 7 & Fall & Closed reduction & No \\
\hline II & Male & 98 & 17 & Atraumatic & Open reduction & No \\
\hline 12 & Male & 69 & 1 & Fall & Closed reduction & Cerebral infarction \\
\hline 13 & Male & 80 & 7 & Atraumatic (BRI) & Closed reduction & No \\
\hline $14 *$ & Female & 88 & 40 & Atraumatic & Open reduction & No \\
\hline 15 & Male & 88 & 38 & Atraumatic (BRI) & Open reduction & No \\
\hline $16 *$ & Female & 91 & 16 & Atraumatic & Open reduction & No \\
\hline 17 & Female & 76 & 30 & Atraumatic & THA & Dementia \\
\hline 18 & Female & 70 & 22 & Atraumatic & Closed reduction & No \\
\hline $19 *$ & Female & 87 & 21 & Atraumatic (BRI) & Open reduction & Dementia \\
\hline $20^{*}$ & Female & 80 & 38 & Fall & Open reduction & No \\
\hline
\end{tabular}

Note: *Dissociation.

Abbreviations: THA, total hip arthroplasty; BRI, bed-related injury.

vertical distance difference, RC to GTT vertical distance, RC to GTT vertical distance difference, RC to GTT horizontal distance and RC to GTT horizontal distance difference. In addition, femoral measurements included residual femoral neck length (RFNL), femoral neck-shaft angle (FNSA), FNSA difference, offset, offset difference and leg length difference (Figure 2). Distance discrepancy, defined as the absolute value of distance difference, was also analyzed.

\section{Statistical Analysis}

Categorical data were presented as absolute numbers and percentages. Continuous data were presented as mean values, standard deviation, and ranges. Independent sample $t$-test and Mann-Whitney $U$-test were used in the analysis of
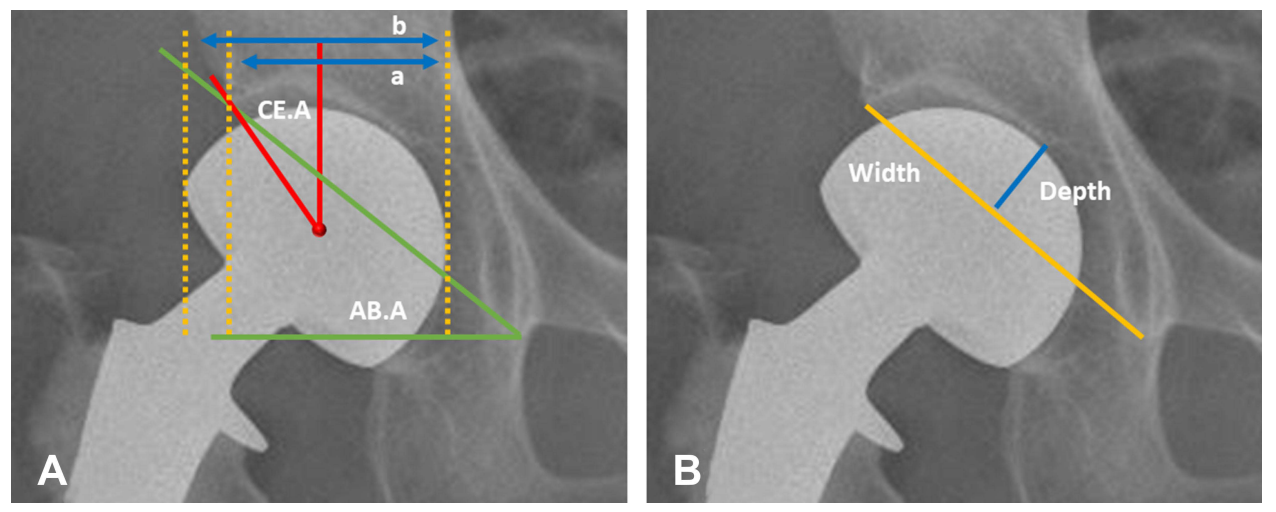

Figure I Acetabular measurements. (A) Measurement of center-edge angle (CE.A), abduction angle (AB.A), Femoral head coverage ratio: ratio of the length between the innermost point of the femoral head and the outer corner of the acetabulum to the length of the femoral head (ratio of a to b). (B) Measurement of acetabular width and acetabular depth. 


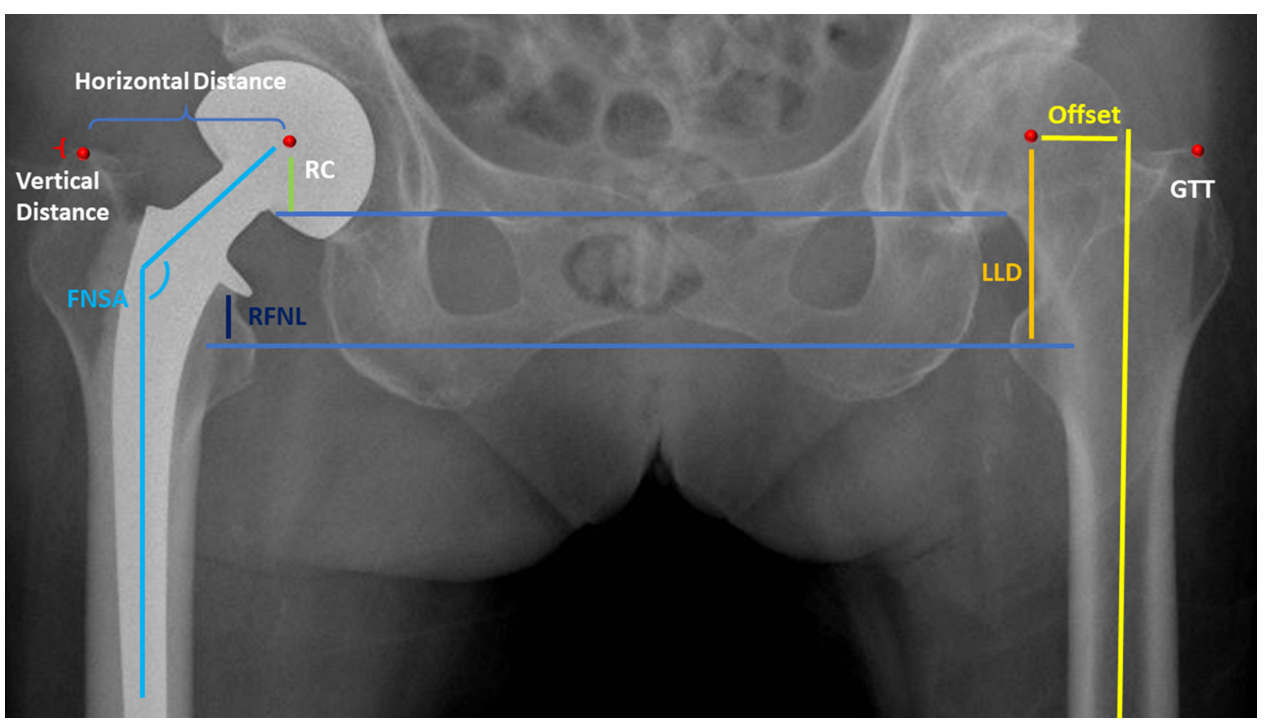

Figure 2 Rotation center measurements and femoral measurements. The interteardrop line was defined as the horizontal reference line. RFNL was the perpendicular distance of a line passing through the medial cortex of femoral osteotomy to the corresponding tip of the lesser trochanter. LLD was the difference in perpendicular distance of a line passing through the RC to the corresponding tip of the lesser trochanter. Offset was the perpendicular distance passing through the RC to the longitudinal axis of the femur.

Abbreviations: RC, rotation center; GTT, greater trochanter tip; RFNL, residual femoral neck length; FNSA, femoral neck-shaft angle; LLD, leg length difference.

quantitative independent data. The Chi-square test was used in the analysis of qualitative independent data. Variables with a $\mathrm{P}<0.10$ in the univariable analysis were considered for the multivariable analysis. Multivariable logistic regression analysis was used for the multivariable analysis. All statistical analyses were conducted using SPSS version 21.0 software (SPSS, Chicago, IL, USA). $\mathrm{P}<0.05$ was considered statistically significant.

For assessing intraobserver reliability, two analysts performed point selection and corresponding measurements blindly and independently. For assessing intra-observer reliability, measurements were repeated one month later by the same analysts. The intraclass correlation coefficient (ICC) was used to calculate interobserver and intra-observer effects. A post hoc power calculation was performed for eliminating type II errors using G-Power 3.1.

\section{Results}

The incidence of dislocation in bipolar hemiarthroplasty was 5.01\% (24 of 479 hips), in which 7 male and 13 female patients met the inclusion criteria in this present study (Figure 3). The mean patient age in the dislocation group was
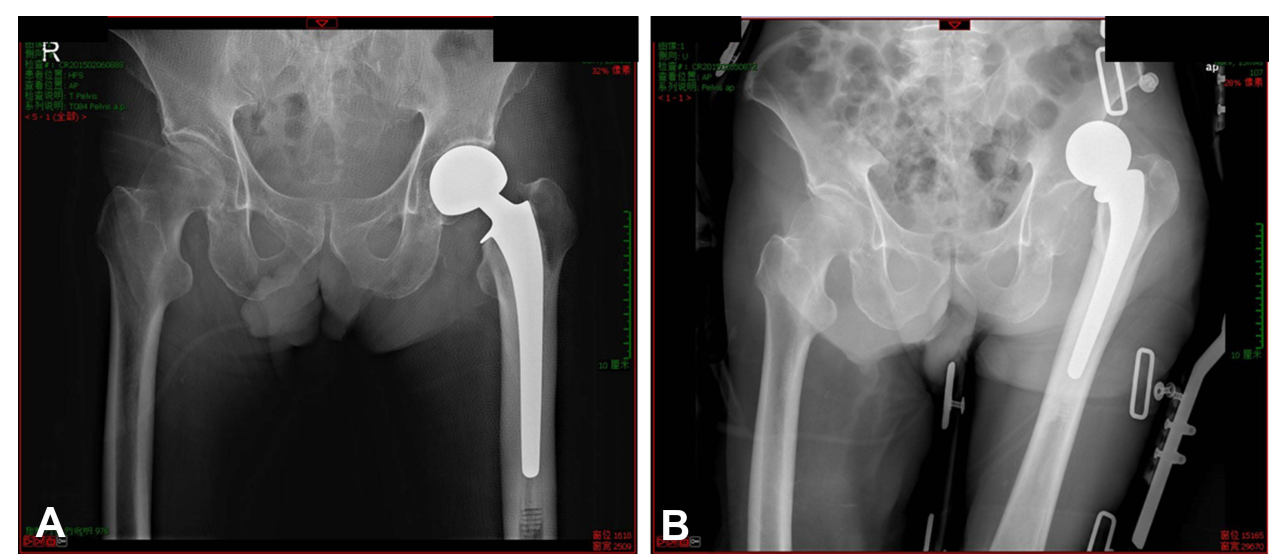

Figure 3 The radiographs of a bipolar hemiarthroplasty dislocation in a80-year-old male patient even with postoperative hip abduction orthosis. (A) One-day postoperative anteroposterior pelvic view; (B) Five- day postoperative anteroposterior pelvic view. 
$80.95 \pm 7.45$ (range, 64-93) years. Among them, five patients suffered from dissociations. The median time from bipolar hemiarthroplasty to the first incident of dislocation was 38.75 (range, 1-340) days. Nineteen dislocations (95\%) occurred within 2 months after surgery and 1 case at 340 days. The mechanism leading to dislocation in 16 patients (80\%) was atraumatic, in which 6 cases suffered from dislocation during the process of getting in or out of bed, and the other four cases $(20 \%)$ were the result of a same-level fall. According to the patient-related factors, no statistically significant difference existed between the two groups regarding operation side, prosthetic type and coexisting disease, including Parkinson's disease, dementia, cerebral infarction. Worth mentioning, the ratio of contralateral hip arthroplasty in the dislocation group was significantly higher than that in the control group (Table 2).

Compared with the matched control group, a statistically significant difference was observed in $\mathrm{CE}$ angle ( $\mathrm{P}<0.001)$, abduction angle $(\mathrm{P}<0.001)$, acetabular depth $(\mathrm{P}=0.002)$, depth/width ratio $(\mathrm{P}=0.002)$, RC to GTT vertical distance $(\mathrm{P}=0.005), \mathrm{RC}$ to GTT vertical distance difference $(\mathrm{P}<0.001)$, $\mathrm{RC}$ to GTT horizontal distance $(\mathrm{P}=0.020)$, RC to GTT horizontal distance difference ( $\mathrm{P}=0.006)$, offset difference $(\mathrm{P}=0.041)$ and offset discrepancy $(\mathrm{P}=0.010)$ (Table 3$)$. Herein, the absolute value of distance difference was also shown as distance discrepancy. Intraclass correlation coefficient results of the intraobserver and interobserver reliabilities ranged from 0.95 to 0.98 and from 0.89 to 0.93 , respectively, which were in the excellent range. Post hoc power analysis revealed a power of $>0.95$ for detecting a significant difference between the dislocation group and control group, except for offset difference (0.78).

Variables with a $\mathrm{P}$ value of $<0.10$ in the univariable analysis were finally included in the multivariable logistic regression analysis. When independent risk factors affecting dislocation were evaluated, decreased $\mathrm{CE}$ angle, increased abduction angle, decreased RC to GTT vertical distance, decreased offset difference and increased offset discrepancy were determined to be independent risk factors of dislocation $(\mathrm{P}=0.025$, OR: 0.706; $\mathrm{P}=0.008$, OR: 1.691; $\mathrm{P}=0.009$, OR: 0.605; P $=0.001$, OR: 0.772; $\mathrm{P}=0.006$, OR: 1.414, respectively) (Table 4).

The proportion of patients who experienced bipolar dissociations was $1.04 \%$ ( 5 of 479 hips), including five women with a mean age of $84.4 \pm 5.5$ years (Figure 4 ). All the patients were successfully treated by open reduction. Among the patients with dissociations, there were one with dementia (25\%), and one with cerebral infarction (25\%). Further, all the implanted femoral heads were smaller than $43 \mathrm{~mm}(100 \%)$. The femoral head size of the dislocation group was significantly smaller than that of the control group $(t=-2.758, \mathrm{P}=0.007)$. Herein, the femoral head size smaller than $43 \mathrm{~mm}$ was the risk factor of dissociation $(\mathrm{OR}=24.38, \mathrm{P}<0.001)$.

\section{Discussion}

The bipolar hemiarthroplasty has been widely used to treat displaced femoral neck fractures in elderly patients, providing pain relief, functional improvement, and low revision rate. ${ }^{7,20}$ Due to the bipolar device, articulating movement is theoretically allowed both at the acetabular-prosthetic interface and within bipolar femoral heads. Thus, dislocation in bipolar hemiarthroplasty is a relatively uncommon but devastating complication. In this present study, decreased CE angle, increased abduction angle, decreased RC to GTT vertical distance, decreased offset difference and increased offset discrepancy were significantly associated with an increased risk of dislocation.

Table 2 Demographic Data

\begin{tabular}{|l|l|l|l|}
\hline & Dislocation (n=20) & Control (n=100) & P value \\
\hline Age (years) (min-max) & $80.95 \pm 7.45$ (64 to 93) & $81.43 \pm 7.15$ (62 to 97) & 0.786 \\
Gender (Male/female) & $7 / 13$ & $33 / 67$ & 0.862 \\
Side (Left/Right) & $4 / 16$ & $5 / 95$ & 0.220 \\
Parkinson's (Yes/No) & $2 / 18$ & $2 / 98$ & 0.069 \\
Dementia (Yes/No) & $3 / 17$ & $8 / 92$ & 0.322 \\
Cerebral infarction (Yes/No) & $2 / 18$ & $14 / 86$ & 0.631 \\
Prosthetic type (Cemented /Cementless) & $13 / 7$ & $69 / 31$ & 0.786 \\
Contralateral hip arthroplasty (Yes/No) & $4 / 16$ & $5 / 95$ & $0.020^{*}$ \\
\hline
\end{tabular}

Notes: Values are expressed as the mean and the standard deviation, with range in parentheses; ${ }^{\mathrm{p}}<0.05$, when compared with control group. 
Table 3 Comparison of Morphologic Measurements Between Dislocation and Control Group

\begin{tabular}{|c|c|c|c|}
\hline & Dislocation (Min-Max) $(n=20)$ & Control (Min-Max) $(n=100)$ & $P$ value \\
\hline \multicolumn{4}{|l|}{ Acetabular Measurements } \\
\hline CE angle $\left({ }^{\circ}\right)$ & $35.25 \pm 6.16$ (25.70 to 45.87$)$ & $43.34 \pm 5.04(36.15$ to 61.61$)$ & $<0.001^{* *}$ \\
\hline Abduction angle $\left({ }^{\circ}\right)$ & $38.17 \pm 3.87$ (28.05 to 43.32$)$ & $33.67 \pm 3.32(23.80$ to 40.80$)$ & $<0.001^{* *}$ \\
\hline Acetabular depth (mm) & $14.72 \pm 2.58$ (9.78 to 18.68$)$ & $16.85 \pm 1.60(12.79$ to $22.3 \mid)$ & $0.002^{* *}$ \\
\hline Acetabular width $(\mathrm{mm})$ & $56.28 \pm 2.79$ (52.30 to 61.39$)$ & $56.57 \pm 3.50$ (48.22 to 64.97$)$ & 0.727 \\
\hline Depth/Width & $0.26 \pm 0.04$ (0.18 to 0.35$)$ & $0.30 \pm 0.03(0.25$ to 0.45$)$ & $0.002 * *$ \\
\hline Coverage ratio & $0.85 \pm 0.06$ (0.76 to 1.00$)$ & $0.90 \pm 0.04$ (0.79 to 1.00$)$ & 0.447 \\
\hline \multicolumn{4}{|l|}{ Rotation Center Measurements } \\
\hline Cup/femoral head diameter difference $(\mathrm{mm})$ & $-0.55 \pm 2.32(-3.74$ to 6.66$)$ & $0.02 \pm 1.45(-4.89$ to 4.62$)$ & 0.164 \\
\hline RC vertical distance difference $(\mathrm{mm})$ & $2.04 \pm 2.31$ ( $(-2.5$ I to 7.65$)$ & $1.47 \pm 2.40(-6.16$ to 7.49$)$ & 0.331 \\
\hline RC to GTT vertical distance $(\mathrm{mm})$ & $-2.58 \pm 5.43(-11.58$ to 9.19$)$ & $1.40 \pm 3.98(-8.35$ to 15.27$)$ & $0.005^{* *}$ \\
\hline RC to GTT vertical distance difference $(\mathrm{mm})$ & $-3.19 \pm 5.74(-20.09$ to 4.04$)$ & I.47 \pm 4.15 (-8.80 to 19.50$)$ & $<0.001^{* *}$ \\
\hline RC to GTT vertical distance discrepancy $(\mathrm{mm})$ & $4.81 \pm 4.39$ (0.58 to 20.09$)$ & $3.31 \pm 2.88(0.01$ to 19.50$)$ & 0.057 \\
\hline RC to GTT horizontal distance $(\mathrm{mm})$ & $35.74 \pm 6.82$ (23.08 to 48.26$)$ & $39.16 \pm 5.7 \mathrm{I}(27.70$ to $58.3 \mathrm{I})$ & $0.020 *$ \\
\hline RC to GTT horizontal distance difference $(\mathrm{mm})$ & $-0.72 \pm 5.59(-11.58$ to 9.19$)$ & $3.32 \pm 5.98(-13.60$ to 19.19$)$ & $0.006 * *$ \\
\hline RC to GTT horizontal distance discrepancy $(\mathrm{mm})$ & $4.75 \pm 2.83$ (0.23 toll.58) & $5.25 \pm 4.36(0.04$ to 19.19$)$ & 0.629 \\
\hline \multicolumn{4}{|l|}{ Femoral Measurements } \\
\hline RFNL (mm) & $13.13 \pm 3.82$ (7.37 to 23.38$)$ & $13.62 \pm 4.48(4.65$ to 25.01$)$ & 0.643 \\
\hline FNSA $\left({ }^{\circ}\right)$ & | $39.73 \pm 9.58$ (|27.59 to |64.56) & $|40.4| \pm 7.5 \mid$ (I26.54 to I58.36) & 0.725 \\
\hline FNSA difference $\left({ }^{\circ}\right)$ & $5.16 \pm 12.16(-26.21$ to 28.40$)$ & $2.55 \pm 9.99(-28.82$ to 24.02$)$ & 0.306 \\
\hline Offset (mm) & $28.07 \pm 6.82(15.82$ to 40.26$)$ & $29.95 \pm 5.54$ (I4.53 to 41.05$)$ & 0.186 \\
\hline Offset difference (mm) & $-3.30 \pm 10.12(-23.68$ to 20.88$)$ & $|.82 \pm 6.7|(-10.7 \mid$ to 20.60$)$ & $0.04 I^{*}$ \\
\hline Offset discrepancy (mm) & $8.32 \pm 6.41$ (0.42 to 23.68$)$ & $5.14 \pm 4.65(0.01$ to 20.60$)$ & $0.010^{*}$ \\
\hline Leg length difference (mm) & $1.93 \pm 5.84(-13.34$ to II.37) & $2.88 \pm 4.33$ (-8.36 to I2.II) & 0.403 \\
\hline Leg length discrepancy (mm) & $4.77 \pm 3.75(0.15$ to 13.34$)$ & $4.15 \pm 3.12(0.02$ to 12.11$)$ & 0.437 \\
\hline
\end{tabular}

Notes: Values are presented as the mean and the standard deviation, with range in parentheses. RC to GTT Difference $=$ values of RC- values of GTT; Discrepancy $=$ absolute value of the difference; Offset and Leg Length Difference/ Discrepancy $=$ value in dislocation group - value in control group; ${ }^{*} p<0.05,{ }^{* *} \mathrm{p}<0.01$, when compared with control group. Variables with $\mathrm{P}<0.1$ were included in multivariate logistic regression analysis.

Abbreviations: CE angle, center edge angle; RC, rotation center; GTT, greater trochanter tip; RFNL, residual femoral neck length; FNSA, femoral neck-shaft angle. 
Table 4 Independent Risk Factors of Dislocation

\begin{tabular}{|l|l|l|}
\hline & OR $(95 \% \mathbf{~ C l})$ & P value \\
\hline CE angle & $0.706(0.521$ to 0.958$)$ & $0.025 *$ \\
Abduction angle & $1.691(1.144$ to 2.500$)$ & $0.008 * *$ \\
RC to GTT vertical distance & $0.605(0.416$ to 0.881$)$ & $0.009 * *$ \\
Offset difference & $0.772(0.661$ to 0.902$)$ & $0.001 * *$ \\
Offset discrepancy & $1.414(1.102$ to 1.813$)$ & $0.006 * *$ \\
\hline
\end{tabular}

Notes: Difference $=$ value in dislocation group - value in control group; Discrepancy $=$ absolute value of the difference; *p $<0.05,{ }^{* *} \mathrm{p}<0.01$.

Abbreviations: CE angle, center edge angle; RC, rotation center; GTT, greater trochanter tip.

Several studies reported that the posterior surgical approach in hemiarthroplasty was associated with an increased risk of dislocation, ${ }^{21-23}$ leading to a higher rate of revision, especially compared with the anterior approach. ${ }^{7}$ However, some studies also emphasized that surgical approach does not influence the dislocation risk in hemiarthroplasty by sufficient soft tissue repair technique. ${ }^{24,25}$ In the present study, all the patients were treated with bipolar hemiarthroplasty with short external rotator tendons and posterior capsule repairment through a posterolateral surgical approach. The incidence of dislocation was 5.01\%, and the mean onset time of dislocation was 38.75 days. The rate and dislocation time in our study were comparable to the previous cohort studies through anterior ${ }^{16,21,23}$ or posterolateral ${ }^{13,26}$ surgical approaches. Recently, the anterior approach was reported to have a higher incidence of early femoral component failure, particular in Dorr A classification. ${ }^{27}$ In combination with operative skill and patient status, optimal surgery approach should be taken into careful consideration by surgeons.

Various risk factors for dislocation related to patients and surgery have been identified. Patients with neuromuscular and cognitive dysfunction are at increased risk of falls and have high rates of dislocation after hemiarthroplasty. ${ }^{28,29} \mathrm{Li}$ et $\mathrm{al}^{16}$ found that dementia and lower Mini-Mental State Examination scores were associated with an increased risk of dislocation. Ninh et $\mathrm{al}^{30}$ revealed a strong association in hemiarthroplasty $(54.5 \%$ versus $18.8 \%$ ) between mental impairment and dislocation rates. However, Suh et $\mathrm{al}^{9}$ observed similar rates of dislocation in patients with or without the neuromuscular disease. Kizkapan et $\mathrm{al}^{13}$ also suggested that neurological disorders such as delirium and dementia do not influence the risk of dislocation. Similarly, we found that comorbidities including Parkinson's, dementia and cerebral infarction did not affect the risk of dislocation in the present study. Further, we observed patients with contralateral hip arthroplasty had a higher risk of dislocation, which may be attributed to the unbalanced femoral offset. Few reports have identified that dislocation is associated with bilateral hip arthroplasty. Similarly, Liu et al ${ }^{31}$ suggested that dislocation is more likely to develop after THA in patients with bilateral pathological hips and might be associated with poor function of the contralateral hips and self-protect ability deficiency.
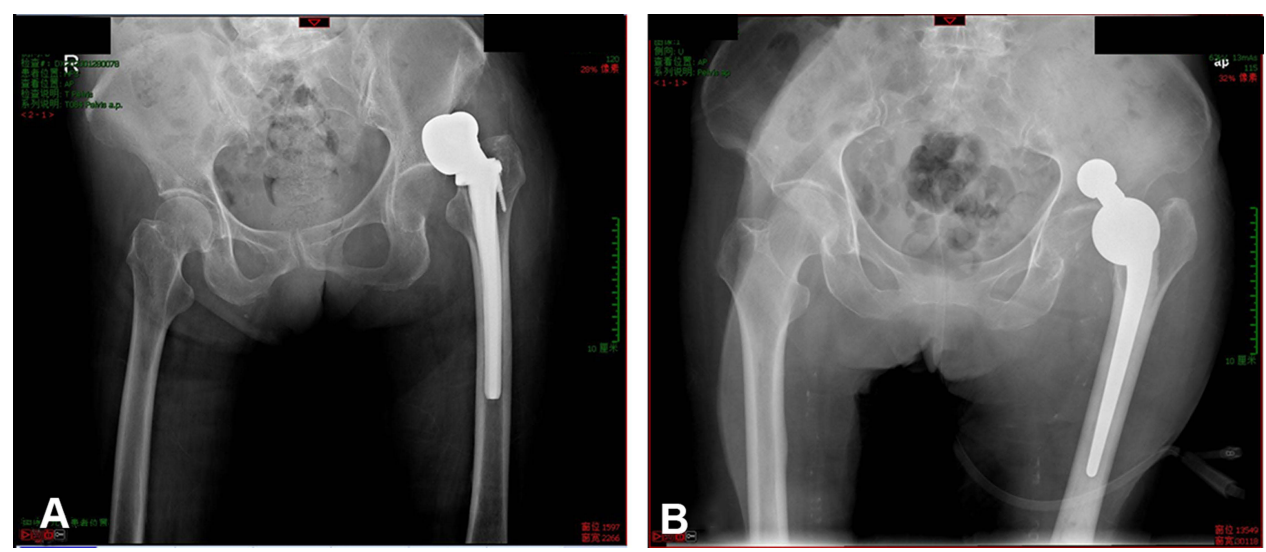

Figure 4 The radiographs of a bipolar hemiarthroplasty dissociation in two female patients.(A) A 80-year-old female patient; (B) A 88-year-old female patient.. 
In the present study, significantly different acetabular factors included $\mathrm{CE}$ angle, abduction angle, acetabular depth, and depth/width ratio. CE angle, determined by the lateral bony coverage, was regarded as an important predictor of the severity of acetabular dysplasia. Decreased CE angle was associated with a shallower and more vertical acetabulum, leading high risk of dislocation. Similarly, Zhang et $\mathrm{al}^{14}$ reported that HA patients with a $\mathrm{CE}$ angle $\leq 45.4^{\circ}$ or an acetabular depth $\leq 19.12 \mathrm{~mm}$ were more likely to suffer dislocation. A Korean study also pointed that defining dislocation as the existence of a $\mathrm{CE}$ angle $>44^{\circ}$ had a sensitivity of $81.3 \%$ and a specificity of $76.0 \%$ in hemiarthroplasty. ${ }^{32}$ Herein, the mean CE angle of the control group was $43.34^{\circ}$, ranging from $36.15^{\circ}$ to $61.61^{\circ}$, indicating the $\mathrm{CE}$ angle limit of dislocation in HA remains further research. Considered that acetabular deficiency could not be figured out in the hemiarthroplasty procedure. Based on the method in this study, preoperative templating contributed to determining the prosthetic size and evaluating the acetabular morphology. According to the patients with acetabular dysplasia, recurrent dislocation usually occurred after two or more times of successful reduction. ${ }^{8}$ Once acetabular dysplasia was recognized, total hip arthroplasty was the optimal selection to reconstruct acetabular offset and maintain hip lateralization (Figure 5).

Few studies referred to the relation between rotation center-related measurements and dislocation. Herein, the position of the greater trochanter tip plays a significant role in affecting the body weight lever arm and hip lateralization. ${ }^{33,34}$ The position of the greater trochanter tip was first evaluated as a risk factor of dislocation. The distance between rotation center and GTT was proven to be reliable in determining femoral offset regardless of the relative position of the femur. ${ }^{35}$ In dislocated group, the significantly decreased RC to GTT distance and distance difference were observed vertically and horizontally. Correspondingly, RC to GTT vertical distance was independently associated with dislocation risk. While in femoral measurements, both offset difference ( $\mathrm{P}=0.001, \mathrm{OR}: 0.772)$ and offset discrepancy ( $\mathrm{P}=0.006$, OR: 1.414) were independently associated with an increased risk of hip dislocation, with no
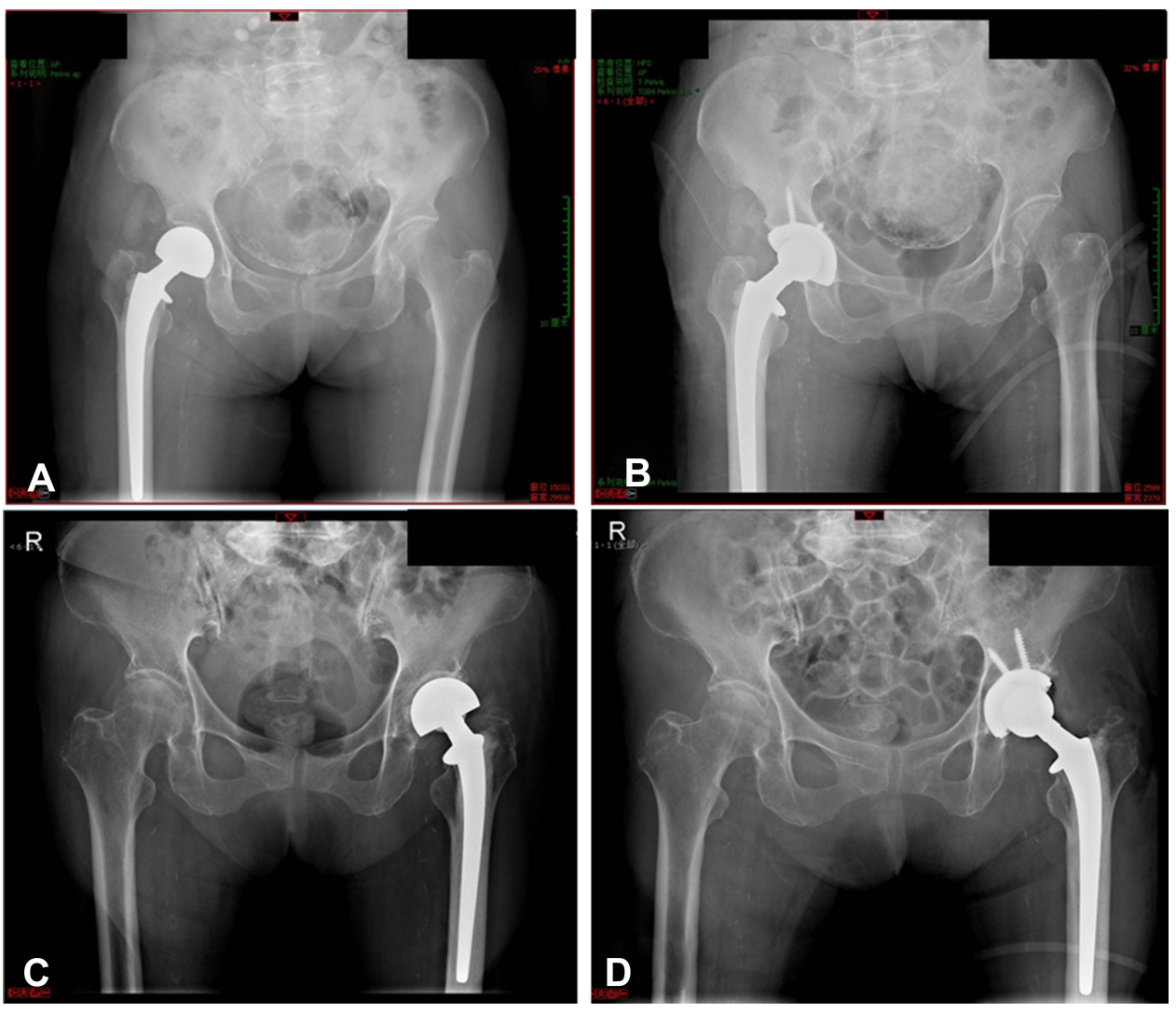

Figure 5 (A) The radiograph of a 92-year-old female patient suffering twice postoperative dislocations with close reduction, whose $\mathrm{CE}$ angle was $27.17^{\circ}$. (B) The postoperative radiograph following revision total hip arthroplasty. (C) The radiograph of an 88-year-old female patient suffering twice postoperative dislocations with once close reduction and once open reduction, whose CE angle was $31.76^{\circ}$. (D) The postoperative radiograph following revision total hip arthroplasty. 
significant difference in femoral offset. Due to the relatively wide variance of femoral offset, the offset difference was better to predict limb equalization and soft tissue tension. Pajarinen et $\mathrm{al}^{23}$ and $\mathrm{Li}$ et $\mathrm{al}^{16}$ arrived at similar conclusions that offset difference instead of offset was associated with postoperative dislocation. Meanwhile, the residual femoral neck length, femoral neck-shaft angle, and leg length difference did not affect the risk of dislocation. Once the existence of dislocation risk was recognized in the simulated templating preoperatively, anterior or anterolateral approach was suggested to reduce risk of dislocation in HA. ${ }^{8,36,37}$

The proportion of patients in our study suffering a dissociation was $1.04 \%$ and all the patients were successfully treated by open reduction. During the surgery, no erosion was detected on the polyethylene cup. Both the polyethylene cup and the head were deeply cleared and we did not revise the components. The hip was stable during the final testing and no further problems were experienced in the postoperative period. Dissociation is rare and corresponding reasons have been reported in only a few case reports. ${ }^{17-19}$ The most common causes were "bottle-opener mechanism" during closed reduction and failure of the polyethylene locking ring. According to the component size, Li et al ${ }^{16}$ reported a rate of $0.9 \%$ (four of 424) patients experiencing dissociations after HA. Among them, two of the four dissociations were in patients with small cups (size $\leq 43 \mathrm{~mm}$ ). Similarly, all the dissociated component sizes in the current study were smaller than $43 \mathrm{~m}$, which had no polyethylene locking ring between shell and inner head according to the implant design in the current study. Accordingly, proximal translation of the affected limb and adduction under fluoroscopic supervision is recommended during reduction maneuvers. ${ }^{38}$ Worth mentioning, the number of dissociations was still too small to perform further statistical analysis of risk factors.

\section{Limitations}

The limitations of our study should be noted. First, this was retrospective study with a relatively small sample size. While the results of statistical analysis indicated reliable reproducibility. Second, though comorbidities including Parkinson's, dementia and cerebral infarction were analyzed, the patient's living status, mental state score and fall risk score were not evaluated. Last, our study population only included patients who underwent hemiarthroplasty through posterolateral approach. The corresponding morphological parameters of dislocation risk might be different through different approaches.

\section{Conclusion}

After the posterolateral approach for treatment of femoral neck fractures using bipolar hemiarthroplasty, 5.01\% of hips dislocated, and 1.04\% experienced dissociation. Decreased CE angle, increased abduction angle, decreased RC to GTT vertical distance, decreased offset difference and increased offset discrepancy were determined to be independent risk factors of HA dislocation. Once dislocation risk was detected by simulated templating, THA or changing surgical approach should be considered to avoid evitable perioperative complications.

\section{Data Sharing Statement}

All the data used and/or analyzed during this study are available upon reasonable request from the corresponding author.

\section{Ethics Approval and Consent to Participate}

This study was approved by the institutional review board of Guangdong Provincial People's Hospital (IRB: 2019664H). All methods were carried out in accordance with relevant guidelines and regulations. Informed consent was obtained in writing from all the individual participants included in the study. We present here a study that complies with the Declaration of Helsinki.

\section{Consent for Publication}

Written informed consent was obtained from all patients for publication of this study and any accompanying images. 


\section{Author Contributions}

All authors made a significant contribution to the work reported, whether that is in the conception, study design, execution, acquisition of data, analysis and interpretation, or in all these areas; took part in drafting, revising or critically reviewing the article; gave final approval of the version to be published; have agreed on the journal to which the article has been submitted; and agree to be accountable for all aspects of the work.

\section{Funding}

This study was supported by Medical Science and Technology Research Fund of Guangdong Province (A2020026), Program of Science and Technology of Guangzhou (202102020959, 202102020336).

\section{Disclosure}

The authors declare that they have no conflicts of interest.

\section{References}

1. Gao L, Han Z, Xiong A. Total hip arthroplasty or hemiarthroplasty for hip fracture. N Engl J Med. 2020;382(11):1072-1073. doi:10.1056/ NEJMc2000062

2. Florschutz AV, Langford JR, Haidukewych GJ, et al. Femoral neck fractures: current management. J Orthop Trauma. 2015;29(3):121-129. doi:10.1097/bot.0000000000000291

3. Ekhtiari S, Gormley J, Axelrod DE, et al. Total hip arthroplasty versus hemiarthroplasty for displaced femoral neck fracture: a systematic review and meta-analysis of randomized controlled trials. J Bone Joint Surg Am. 2020;102(18):1638-1645. doi:10.2106/jbjs.20.00226

4. Ravi B, Pincus D, Khan H, et al. Comparing complications and costs of total hip arthroplasty and hemiarthroplasty for femoral neck fractures: a propensity score-matched, population-based study. J Bone Joint Surg Am. 2019;101(7):572-579. doi:10.2106/jbjs.18.00539

5. Lee BP, Berry DJ, Harmsen WS, et al. Total hip arthroplasty for the treatment of an acute fracture of the femoral neck: long-term results. $J$ Bone Joint Surg Am. 1998;80(1):70-75. doi:10.2106/00004623-199801000-00012

6. Burgers PT, Van Geene AR, Van den Bekerom MP, et al. Total hip arthroplasty versus hemiarthroplasty for displaced femoral neck fractures in the healthy elderly: a meta-analysis and systematic review of randomized trials. Int Orthop. 2012;36(8):1549-1560. doi:10.1007/s00264-012-1569-7

7. Moerman S, Mathijssen NMC, Tuinebreijer WE, et al. Hemiarthroplasty and total hip arthroplasty in 30,830 patients with hip fractures: data from the Dutch Arthroplasty Register on revision and risk factors for revision. Acta Orthop. 2018;89(5):509-514. doi:10.1080/ 17453674.2018.1499069

8. Gill JR, Kiliyanpilakkill B, Parker MJ. Management and outcome of the dislocated hip hemiarthroplasty. Bone Joint J. 2018;100-b(12):1618-1625. doi:10.1302/0301-620x.100b12.Bjj-2018-0281.R1

9. Suh KT, Kim DW, Lee HS, et al. Is the dislocation rate higher after bipolar hemiarthroplasty in patients with neuromuscular diseases? Clin Orthop Relat Res. 2012;470(4):1158-1164. doi:10.1007/s11999-011-2139-9

10. Salem KM, Shannak OA, Scammell BE, et al. Predictors and outcomes of treatment in hip hemiarthroplasty dislocation. Ann R Coll Surg Engl. 2014;96(6):446-451. doi:10.1308/003588414x13946184903045

11. White SM, Griffiths R. Projected incidence of proximal femoral fracture in England: a report from the NHS Hip Fracture Anaesthesia Network (HIPFAN). Injury. 2011;42(11):1230-1233. doi:10.1016/j.injury.2010.11.010

12. Mukka S, Lindqvist J, Peyda S, et al. Dislocation of bipolar hip hemiarthroplasty through a postero-lateral approach for femoral neck fractures: a cohort study. Int Orthop. 2015;39(7):1277-1282. doi:10.1007/s00264-014-2642-1

13. Kizkapan TB, Misir A, Uzun E, et al. Factors affecting dislocation after bipolar hemiarthroplasty in patients with femoral neck fracture. Injury. 2020;51(3):663-669. doi:10.1016/j.injury.2020.01.025

14. Zhang Y, Yao Z, Shi P, et al. Morphological risk factors associated with dislocation after bipolar hemiarthroplasty of the hip in patients with femoral neck fractures-a nested case-control study. J Orthop Surg Res. 2019;14(1):395. doi:10.1186/s13018-019-1409-1

15. Jones C, Briffa N, Jacob J, et al. The Dislocated Hip Hemiarthroplasty: current Concepts of Etiological factors and Management. Open Orthop J. 2017;11:1200-1212. doi:10.2174/1874325001711011200

16. Li L, Ren J, Liu J, et al. What are the risk factors for dislocation of hip bipolar hemiarthroplasty through the anterolateral approach? A nested case-control study. Clin Orthop Relat Res. 2016;474(12):2622-2629. doi:10.1007/s11999-016-5053-3

17. Uruç V, Özden R, Duman İG, et al. Five cases of early dissociation between the bipolar hip endoprosthesis cup components; either spontaneously or during reduction maneuvers. Acta Orthop Traumatol Turc. 2017;51(2):172-176. doi:10.1016/j.aott.2016.12.007

18. Grauke LJ, Richardson ML. Dissociation of a bipolar prosthesis after right hip hemiarthroplasty. Radiol Case Rep. 2006;1(4):123-125. doi:10.2484/ rcr.v1i4.55

19. Yuenyongviwat V, Iamthanaporn K, Hongnaparak T. Spontaneous dissociation of bipolar hip hemiarthroplasty in a patient with nerve palsy: a case report and review of the literature. Int J Surg Case Rep. 2015;15:112-115. doi:10.1016/j.ijscr.2015.08.034

20. Rogmark C, Leonardsson O. Hip arthroplasty for the treatment of displaced fractures of the femoral neck in elderly patients. Bone Joint J. 2016;98b(3):291-297. doi:10.1302/0301-620x.98b3.36515

21. Enocson A, Tidermark J, Tornkvist H, et al. Dislocation of hemiarthroplasty after femoral neck fracture: better outcome after the anterolateral approach in a prospective cohort study on 739 consecutive hips. Acta Orthop. 2008;79(2):211-217. doi:10.1080/17453670710014996

22. Rogmark C, Fenstad AM, Leonardsson O, et al. Posterior approach and uncemented stems increases the risk of reoperation after hemiarthroplasties in elderly hip fracture patients. Acta Orthop. 2014;85(1):18-25. doi:10.3109/17453674.2014.885356 
23. Pajarinen J, Savolainen V, Tulikoura I, et al. Factors predisposing to dislocation of the Thompson hemiarthroplasty: 22 dislocations in 338 patients. Acta Orthop Scand. 2003;74(1):45-48. doi:10.1080/00016470310013644

24. Wallner O, Stark A, Muren O, et al. Unstable hip arthroplasties. A prospective cohort study on seventy dislocating hips followed up for four years. Int Orthop. 2015;39(6):1037-1044. doi:10.1007/s00264-014-2583-8

25. Ko CK, Law SW, Chiu KH. Enhanced soft tissue repair using locking loop stitch after posterior approach for hip hemiarthroplasty. $J$ Arthroplasty. 2001;16(2):207-211. doi:10.1054/arth.2001.20539

26. Madanat R, Mäkinen TJ, Ovaska MT, et al. Dislocation of hip hemiarthroplasty following posterolateral surgical approach: a nested case-control study. Int Orthop. 2012;36(5):935-940. doi:10.1007/s00264-011-1353-0

27. Angerame MR, Fehring TK, Masonis JL, et al. Early failure of primary total hip arthroplasty: is surgical approach a risk factor? $J$ Arthroplasty. 2018;33(6):1780-1785. doi:10.1016/j.arth.2018.01.014

28. Turcotte R, Godin C, Duchesne R, et al. Hip fractures and Parkinson's disease. A clinical review of 94 fractures treated surgically. Clin Orthop Relat Res. 1990;256:132-136. doi:10.1097/00003086-199007000-00020

29. Sierra RJ, Schleck CD, Cabanela ME. Dislocation of bipolar hemiarthroplasty: rate, contributing factors, and outcome. Clin Orthop Relat Res. 2006;442:230-238. doi:10.1097/01.blo.0000183741.96610.c3

30. Ninh CC, Sethi A, Hatahet M, et al. Hip dislocation after modular unipolar hemiarthroplasty. J Arthroplasty. 2009;24(5):768-774. doi:10.1016/j. arth.2008.02.019

31. Liu Q, Cheng X, Yan D, et al. Plain radiography findings to predict dislocation after total hip arthroplasty. J Orthop Transl. 2019;18:1-6. doi:10.1016/j.jot.2018.12.003

32. Kim Y, Kim JK, Joo IH, et al. Risk factors associated with dislocation after bipolar hemiarthroplasty in elderly patients with femoral neck fracture. Hip Pelvis. 2016;28(2):104-111. doi:10.5371/hp.2016.28.2.104

33. Miller TM, Mandell DT, Dannenbaum JH, et al. Anatomic and patient risk factors for postoperative periprosthetic hip fractures: a case-control study. J Arthroplasty. 2020;35(6):1708-1711. doi:10.1016/j.arth.2020.02.007

34. Theivendran K, Hart WJ. Is the tip of the greater trochanter a reliable reference for the rotation centre of the femoral head in total hip arthroplasty? Acta Orthop Belg. 2009;75(4):472-476.

35. Bonin N, Jacquot L, Boulard L, et al. How to best measure femoral length and lateralisation after total hip arthroplasty on antero-posterior pelvic radiographs. Int Orthop. 2016;40(12):2479-2485. doi:10.1007/s00264-016-3145-z

36. Kunkel ST, Sabatino MJ, Kang R, et al. A systematic review and meta-analysis of the direct anterior approach for hemiarthroplasty for femoral neck fracture. Eur J Orthop Surg Traumatol. 2018;28(2):217-232. doi:10.1007/s00590-017-2033-6

37. Abram SG, Murray JB. Outcomes of 807 Thompson hip hemiarthroplasty procedures and the effect of surgical approach on dislocation rates. Injury. 2015;46(6):1013-1017. doi:10.1016/j.injury.2014.12.016

38. Bian YY, Wang LC, Xiao K, et al. Hip dislocation and femoral component disassembly after bipolar hemiarthroplasty: a report of four cases and introduction of new reduction maneuvers. Chin Med J. 2019;132(3):370-372. doi:10.1097/cm9.0000000000000057

Therapeutics and Clinical Risk Management

Dovepress

\section{Publish your work in this journal}

Therapeutics and Clinical Risk Management is an international, peer-reviewed journal of clinical therapeutics and risk management, focusing on concise rapid reporting of clinical studies in all therapeutic areas, outcomes, safety, and programs for the effective, safe, and sustained use of medicines. This journal is indexed on PubMed Central, CAS, EMBase, Scopus and the Elsevier Bibliographic databases. The manuscript management system is completely online and includes a very quick and fair peer-review system, which is all easy to use. Visit http://www. dovepress.com/testimonials.php to read real quotes from published authors.

Submit your manuscript here: https://www.dovepress.com/therapeutics-and-clinical-risk-management-journal 